\title{
Predictors of operating room extubation in adult cardiac surgery
}

Kathirvel Subramaniam, MD, MPH, ${ }^{a}$ Diana S. DeAndrade, MD, ${ }^{a}$ Daniel R. Mandell, MD,

Andrew D. Althouse, PhD, ${ }^{\mathrm{b}}$ Rajan Manmohan, BS, ${ }^{\mathrm{c}}$ Stephen A. Esper, MD, MBA, ${ }^{\mathrm{a}}$

Jeffrey M. Varga, MD, ${ }^{\mathrm{a}}$ and Vinay Badhwar, $\mathrm{MD}^{\mathrm{d}}$

\section{ABSTRACT}

Objective: The primary objective of the study was to identify perioperative factors associated with successful immediate extubation in the operating room after adult cardiac surgery. The secondary objective was to derive a simplified predictive scoring system to guide clinicians in operating room extubation.

Methods: All 1518 patients in this retrospective cohort study underwent standardized fast-track cardiac anesthetic protocol during adult cardiac surgery. Perioperative variables between patients who had successful extubation in the operating room versus in the intensive care unit were retrospectively analyzed using both univariate and multivariable logistic regression analyses. A predictive score of successful operating room extubation was constructed from the multivariable results of 800 patients (derivation set), and the scoring system was further tested using a validation set of 398 patients.

Results: Younger age, lower body mass index, higher preoperative serum albumin, absence of chronic lung disease and diabetes, less-invasive surgical approach, isolated coronary bypass surgery, elective surgery, and lower doses of intraoperative intravenous fentanyl were independently associated with higher probability of operating room extubation. The extubation prediction score created in a derivation set of patients performed well in the validation set. Patient scores less than 0 had a minimal probability of successful operating room extubation. Operating room extubation was highly predicted with scores of 5 or greater.

Conclusions: Perioperative factors that are independently associated with successful operating room extubation after adult cardiac operations were identified, and an operating room extubation prediction scoring system was validated. This scoring system may be used to guide safe operating room extubation after cardiac operations. (J Thorac Cardiovasc Surg 2017;154:1656-65)

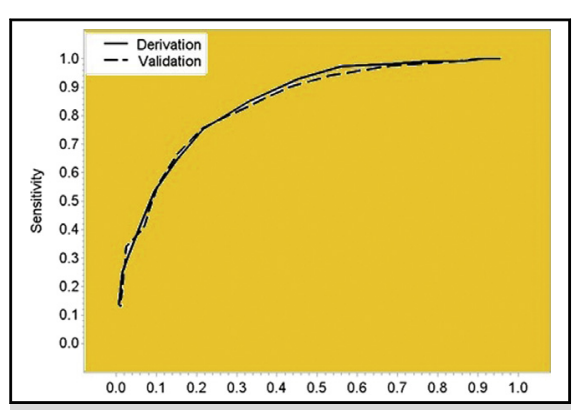

Percentage of patients successfully extubated in the $\mathrm{OR}$ in a validation set.

\section{Central Message}

Independent factors associated with successful OR extubation after cardiac operations were identified, and an extubation prediction score is introduced.

\section{Perspective}

Extubation in the OR after cardiac surgery is feasible and safe in selected patients. In this study, factors were identified that were independently associated with successful OR extubation after cardiac operations. A prediction score was created and validated to guide safe OR extubation practice in patients undergoing cardiac surgery.

See Editorial Commentary page 1666.

See Editorial page 1654.
It is well established that early extubation improves the quality of patient recovery and decreases resource use and healthcare costs. ${ }^{1-5}$ Early extubation is not associated with increased postoperative complication rates after cardiac surgery. ${ }^{6-10}$ Despite these developments, "fast-track"

From the ${ }^{\mathrm{a}}$ Department of Anesthesiology, and ${ }^{\mathrm{b}}$ Heart and Vascular Institute, University of Pittsburgh Medical Center; ${ }^{c}$ University of Pittsburgh School of Medicine, Pittsburgh, Pa; and ${ }^{\mathrm{d}}$ Department of Cardiovascular and Thoracic Surgery, West Virginia University, Morgantown, WVa.

Received for publication July 30, 2016; revisions received May 11, 2017; accepted for publication May 30, 2017; available ahead of print July 12, 2017.

Address for reprints: Kathirvel Subramaniam, MD, MPH, Department of Anesthesiology, University of Pittsburgh, 200 Lothrop St, Pittsburgh, PA 15213 (E-mail: subramaniamk@upmc.edu).

$0022-5223 / \$ 36.00$

Copyright (c) 2017 by The American Association for Thoracic Surgery http://dx.doi.org/10.1016/j.jtcvs.2017.05.107 extubation after cardiac surgery remains globally poorly defined, with protocols for early extubation ranging from 6 or less hours to 24 hours postoperatively. ${ }^{1,6,10-12}$ Prior investigations to determine predictive risk factors for prolonged postoperative mechanical ventilation have largely focused on extubation after transfer to the intensive care unit (ICU). ${ }^{13-17}$ Immediate extubation in the operating room (OR) after cardiac surgery remains a

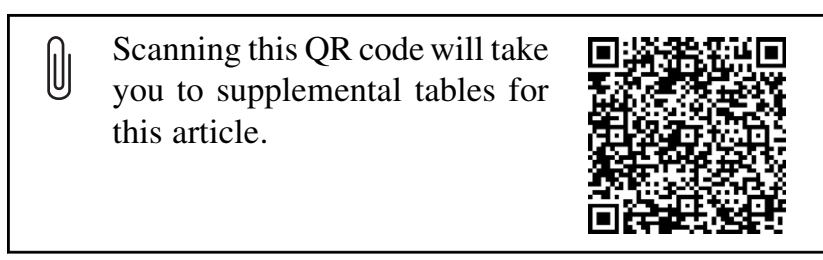



Abbreviations and Acronyms
$\mathrm{CABG}=$ coronary artery bypass grafting
CLD = chronic lung disease
$\mathrm{CPB}=$ cardiopulmonary bypass
ICU = intensive care unit
MVRR $=$ mitral valve repair/replacement
$\mathrm{OR} \quad=$ operating room
STS $=$ Society of Thoracic Surgeons

controversial and infrequent practice, often reserved for highly limited patient populations and procedures. ${ }^{18-21}$ Recent experience has demonstrated the safety and efficacy of OR extubation in adult cardiac operations, with significant cost-effective outcome improvements. ${ }^{22}$

The objectives of this study were to (1) identify preoperative and intraoperative characteristics associated with successful immediate extubation in the OR after adult cardiac operations and (2) create an OR extubation prediction score to guide safe OR extubation.

\section{MATERIALS AND METHODS}

This retrospective cohort study was reviewed by the institutional Quality Improvement Review Committee and approved as a Quality Improvement Project, and waiver of consent was granted. All preoperative and operative variables were collected without exclusion from our institutional Society of Thoracic Surgeons (STS) database version 2.73. Additional intraoperative anesthetic and procedural variables not otherwise recorded by the STS database were obtained from the electronic anesthesia record.

\section{Patient Population}

A total of 1518 patients (aged $>18$ years) underwent cardiac operations between January 1, 2012, and June 30, 2014, at a single academic institution. Before January of 2012, OR extubation was limited to minimally invasive procedures. OR extubation protocols were implemented for all nonemergency open cardiac surgical procedures commencing in January of 2012. An electronic medical recording system for anesthesia documentation also became fully functional in January of 2012. This cohort study was planned and approved as a quality-improvement project in 2014 to study patients undergoing cardiac surgery between January of 2012 and June of 2014

Patients meeting the following clinical criteria were excluded: emergency status, cardiogenic shock, acute bacterial endocarditis, deep hypothermic circulatory arrest, cardiac transplantation, ventricular assist device placement, or transcatheter aortic valve replacement. One patient was excluded because of an incomplete electronic anesthesia record. Urgent status and all other operations, including reoperations, were included. The final study cohort included 1198 patients. A Consolidated Standards of Reporting Trials flow diagram illustrating patient inclusion and exclusion is provided in Figure 1.

\section{Anesthesia and Extubation Protocol}

All patients in the study cohort underwent a standardized fast-track cardiac anesthetic protocol for induction, maintenance, and emergence. ${ }^{22}$ Informed consent for anesthesia was obtained preoperatively from all patients. A dose of intravenous midazolam ranging from 1 to $2 \mathrm{mg}$ was administered in the preoperative holding area or on arrival to the OR. Patients undergoing a thoracotomy approach received intrathecal morphine
0.2 to $0.5 \mathrm{mg}$ for postoperative analgesia provided there were no contraindications to central neuraxial blocks (eg, therapeutic anticoagulation). Intrathecal analgesia was administered immediately before anesthetic induction with the patient in the sitting position after confirmation of cerebrospinal fluid aspiration through a 22- or 25-gauge spinal needle.

After the placement of intrathecal morphine, but before induction, all patients were placed in the supine position, American Society of Anesthesiologists standard monitors were applied, and an invasive arterial line was inserted using local anesthesia. Anesthetic induction medications (propofol, etomidate, ketamine, and midazolam) and their doses were individualized according to the patient's age, weight, and associated comorbid conditions. A limited dose of intravenous fentanyl (50-100 $\mu \mathrm{g})$ was administered during induction of anesthesia. Additional fentanyl was administered during incision, sternotomy, and aortic cannulation as required. Hemodynamics and bispectral index were used to guide anesthetic administration. Protocolized dose ranges of commonly administered anesthesia medications in our fast-track protocol are shown in Table E1. Dose ranges served only as guidelines, and anesthesiologists were allowed to administer additional doses of drugs (midazolam and fentanyl) beyond the listed range per their clinical judgment. The extent of neuromuscular blockade was monitored closely using a peripheral nerve stimulator in the periorbital area throughout the course of the procedure, with the intent to reverse the blockade on completion of surgery.

On reversal of neuromuscular blockade, all patients were assessed for appropriateness of extubation in the OR before ICU transfer. Extubation guidelines for cardiac surgery included (1) hemodynamic stability with minimal vasopressor or inotropic support; (2) awake and able to follow simple commands; (3) adequate muscle strength and respiratory effort; (4) vital capacity greater than $25 \mathrm{~mL} / \mathrm{kg}$; (5) tidal volume greater than $5 \mathrm{~mL} / \mathrm{kg}$; (6) end tidal $\mathrm{CO}_{2}$ less than $50 \mathrm{~mm} \mathrm{Hg}$; (7) no significant metabolic acidosis; (8) normothermia; and (9) absence of significant mediastinal bleeding or coagulopathy. A Standard Wright Spirometer was not used for measuring vital capacity. Assessment of vital capacity was simply done by asking the patient to take a deep breath and measuring the maximum tidal volume on the anesthesia machine. Patients not fulfilling these criteria were given low-dose propofol or dexmedetomidine sedation for the duration of ICU transport.

After an initial ICU assessment, weaning from mechanical ventilation was initiated according to an institutional nursing and respiratory therapy-driven fast-track ICU extubation protocol, with a goal of extubation within 4 hours of ICU arrival. Extubation criteria similar to those used in the OR were used in the ICU. Postoperative analgesia was provided with acetaminophen, limited intravenous opioid analgesics, or oral oxycodone as appropriate.

\section{Data Collection and Statistical Analysis}

Preoperative variables were collected retrospectively from our institutional STS version 2.73 adult cardiac surgery database for all 1198 patients. Intraoperative clinical and anesthetic procedural variables were collected from the electronic anesthesia record. For descriptive purposes, preoperative and intraoperative characteristics are summarized as median (range) for continuous variables and frequency $(\%)$ for categoric variables (Tables 1 and 2). Comparisons between patients extubated successfully in the OR versus those extubated in the ICU were performed using Wilcoxon rank-sum tests for continuous variables and chi-square tests for categoric variables.

Multivariable logistic regression was used to develop 2 prediction scores for successful OR extubation. The first model uses only preoperative patient characteristics (all variables listed in Table 1), whereas the second model includes both preoperative and intraoperative characteristics (the same variables in Table 1 plus all those listed in Table 2). For model derivation, 800 patients were randomly selected and assigned to the model derivation set; the remaining 398 patients were assigned to the model validation set. On the derivation set of 800 patients, we performed 


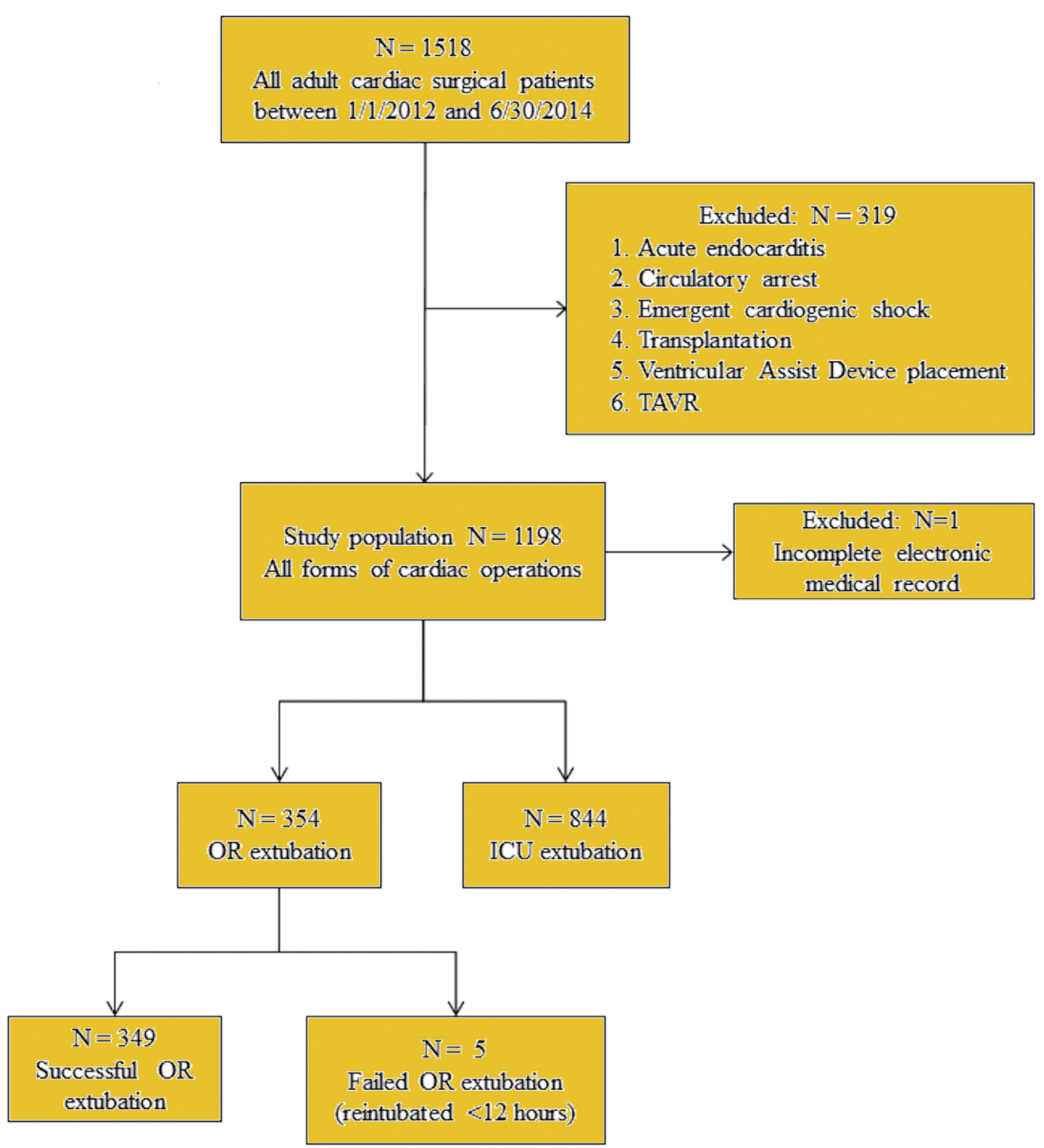

FIGURE 1. Flow diagram of study population characteristics. TAVR, Transcatheter aortic valve replacement; $O R$, operating room; $I C U$, intensive care unit.

multivariable logistic regression with a forward stepwise selection algorithm; independent variables were entered into the model if they were found to have a $P$ value less than .10 on multivariable analysis, with $P$ less than .10 also the threshold to remain in the prediction model at subsequent steps. Because of the rigorous data collection in the STS Adult Cardiac Surgery Database, missing data were rare $(<0.5 \%$ of data points were missing). We used mean imputation for variables with small amounts of missing data $(<5 \%$ of patients); any variables with missing values for more than $5 \%$ of patients were eliminated from consideration for multivariable analysis. In the model(s), a positive $\beta$-coefficient (odds ratio $>1$ ) indicates a characteristic that is associated with higher odds of successful OR extubation, whereas a negative $\beta$-coefficient (odds ratio $<1$ ) indicates a characteristic that is associated with lower odds of successful OR extubation. The $\beta$-coefficients from the predictive model in the derivation set were used to assign points for the OR extubation score; point assignment was carried out using an algorithm based on the $\beta$-coefficients for each risk factor, previously described by the Framingham Heart Study investigators. ${ }^{23}$ The points associated with each risk factor were then added to create an aggregate risk score. The model-based score derived from the derivation set was then applied to the validation set to assess the performance of the model on novel cases; the predictive ability of scoring system was assessed using c-statistics, and the c-statistics of different models were compared using likelihood ratio test. All statistical analyses were conducted using SAS version 9.4 (SAS Institute, Inc, Cary, NC).

\section{RESULTS}

Preoperative patient characteristics (Table 1) and intraoperative variables (Table 2) are summarized between the 2 groups (successful OR extubation vs ICU extubation). Many preoperative factors and intraoperative variables were statistically different between the 2 groups. Multivariate logistic regression analysis was carried out on the derivation set of 800 patients to identify the predictors of successful OR extubation and create an OR extubation 
TABLE 1. Preoperative characteristics of study population

\begin{tabular}{|c|c|c|c|}
\hline Preoperative patient characteristics & ICU extubation $(\mathrm{N}=849)$ & Successful OR extubation $(N=349)$ & $P$ value \\
\hline Male gender* & $529(62 \%)$ & $226(65 \%)$ & .425 \\
\hline Age (y) & $67.0(23-94)$ & $61.0(20-89)$ & $<.001$ \\
\hline BMI $\left(\mathrm{kg} / \mathrm{m}^{2}\right)$ & $28.7(15.6-66.6)$ & $26.9(13.5-44.8)$ & $<.001$ \\
\hline Redo cardiac surgery & $127(15 \%)$ & $29(8 \%)$ & .002 \\
\hline $\begin{array}{l}\text { Status } \\
\quad \text { Elective } \\
\text { Urgent }\end{array}$ & $\begin{array}{l}422(50 \%) \\
427(50 \%)\end{array}$ & $\begin{array}{r}266(76 \%) \\
83(24 \%)\end{array}$ & $<.001$ \\
\hline Preoperative hematocrit $(\%)$ & $37.7(22.9-52)$ & $40.3(13.5-50.4)$ & $<.001$ \\
\hline Preoperative $\mathrm{HbA}_{1} \mathrm{C}(\%)$ & $6.0(4.3-12.6)$ & $5.7(4.3-12.8)$ & $<.001$ \\
\hline Preoperative total albumin $(\mathrm{g} / \mathrm{dL})$ & $3.7(1.3-5)$ & $4.1(2.7-4.9)$ & $<.001$ \\
\hline Preoperative MELD score & $8.4(1-38)$ & $7.5(1-23.3)$ & $<.001$ \\
\hline Preoperative beta-blocker & $702(83 \%)$ & $226(65 \%)$ & $<.001$ \\
\hline Preoperative ACE or ARB & $259(31 \%)$ & $61(18 \%)$ & $<.001$ \\
\hline Preoperative nitrates & $46(5 \%)$ & $11(3 \%)$ & .094 \\
\hline Preoperative inotropes & $44(5 \%)$ & $5(1 \%)$ & .003 \\
\hline Preoperative aspirin & $588(69 \%)$ & $179(51 \%)$ & $<.001$ \\
\hline Preoperative lipid lowering & $480(57 \%)$ & $144(41 \%)$ & $<.001$ \\
\hline Preoperative ADP inhibitor & $78(9 \%)$ & $14(4 \%)$ & .002 \\
\hline Smoking history & $153(18 \%)$ & $73(21 \%)$ & .244 \\
\hline Diabetes & $357(42 \%)$ & $73(21 \%)$ & $<.001$ \\
\hline Dyslipidemia & $673(79 \%)$ & $232(67 \%)$ & $<.001$ \\
\hline Dialysis & $26(3 \%)$ & $6(2 \%)$ & .190 \\
\hline Hypertension & $763(90 \%)$ & $259(74 \%)$ & $<.001$ \\
\hline Cerebrovascular disease & $178(21 \%)$ & $46(13 \%)$ & .002 \\
\hline Prior CVA & $80(9 \%)$ & $22(6 \%)$ & .726 \\
\hline Prior transient ischemic attack & $73(9 \%)$ & $21(6 \%)$ & .570 \\
\hline CLD & $118(14 \%)$ & $16(5 \%)$ & $<.001$ \\
\hline Home oxygen & $46(5 \%)$ & $3(1 \%)$ & $<.001$ \\
\hline Sleep apnea & $173(20 \%)$ & $41(12 \%)$ & $<.001$ \\
\hline Recent preoperative pneumonia & $42(5 \%)$ & $6(2 \%)$ & .032 \\
\hline Peripheral vascular disease & $192(23 \%)$ & $43(12 \%)$ & $<.001$ \\
\hline Alcohol $>7$ drinks/wk & $81(10 \%)$ & $51(15 \%)$ & .001 \\
\hline Cancer & $60(7 \%)$ & $20(6 \%)$ & .400 \\
\hline Prior mediastinal radiation & $42(5 \%)$ & $6(2 \%)$ & .010 \\
\hline Prior pacemaker & $63(7 \%)$ & $12(3 \%)$ & .064 \\
\hline Prior AICD & $30(4 \%)$ & $6(2 \%)$ & .261 \\
\hline Prior MI & $298(35 \%)$ & $82(24 \%)$ & $<.001$ \\
\hline $\begin{array}{l}\text { MI timing } \\
\quad<6 \mathrm{~h} \\
6-24 \mathrm{~h} \\
1-7 \mathrm{~d} \\
8-21 \mathrm{~d}\end{array}$ & $\begin{array}{c}3(0.4 \%) \\
98(12 \%) \\
53(6 \%) \\
144(17.0 \%)\end{array}$ & $\begin{array}{c}1(0.3 \%) \\
25(7 \%) \\
6(2 \%) \\
50(14 \%)\end{array}$ & .082 \\
\hline Preoperative NYHA class III/IV & $339(40 \%)$ & $79(23 \%)$ & $<.001$ \\
\hline $\begin{array}{l}\text { Preoperative angina } \\
\text { Class III } \\
\text { Class IV }\end{array}$ & $\begin{array}{l}146(17 \%) \\
144(17 \%)\end{array}$ & $\begin{array}{l}54(16 \%) \\
34(10 \%)\end{array}$ & .014 \\
\hline
\end{tabular}


TABLE 1. Continued

\begin{tabular}{|c|c|c|c|}
\hline Preoperative patient characteristics & ICU extubation $(\mathrm{N}=849)$ & Successful OR extubation $(\mathrm{N}=349)$ & $P$ value \\
\hline Presentation & & & .004 \\
\hline No symptoms or unlikely to be ischemia & $480(57 \%)$ & $231(66 \%)$ & \\
\hline Stable angina & $61(7 \%)$ & $26(7 \%)$ & \\
\hline Unstable angina or MI & $308(36 \%)$ & $92(26 \%)$ & \\
\hline Preoperative cardiogenic shock & $5(1 \%)$ & $0(0.0 \%)$ & .150 \\
\hline Preoperative resuscitation & $0(0.0 \%)$ & $0(0.0 \%)$ & NA \\
\hline Preoperative ventricular tachycardia or ventricular fibrillation & $15(2 \%)$ & $5(1 \%)$ & .472 \\
\hline Preoperative LVEF (\%) & $55(10-80)$ & $55(5-70)$ & $<.001$ \\
\hline \multicolumn{4}{|l|}{ STS risk scores } \\
\hline PROM & $1.9(0.2-34.7)$ & $0.7(0.2-31.7)$ & $<.001$ \\
\hline Prolonged ventilator requirement & $10.1(2.3-77.6)$ & $4.9(2-60.1)$ & $<.001$ \\
\hline PRMM & $16.0(3.8-82)$ & $9.3(3.6-68.1)$ & $<.001$ \\
\hline
\end{tabular}

$\overline{I C U}$, Intensive care unit; $O R$, operating room; $B M I$, body mass index; $H b A_{l} C$, glycated hemoglobin; $M E L D$, Model for End-stage Liver Disease; $A C E$, angiotensin-converting enzyme; $A R B$, angiotensin receptor blocker; $A D P$, adenosine diphosphate; $C V A$, cerebrovascular accident; $C L D$, chronic lung disease; $A I C D$, automatic implantable cardioverter defibrillator; MI, myocardial infarction; NYHA, New York Heart Association; NA, not available; LVEF, left ventricular ejection fraction; STS, Society of Thoracic Surgeons; PROM, Predicted Risk of Mortality; PRMM, predicted risk of mortality and morbidity. *Note that the percentages in this table are column percentages, eg, 226 of 349 patients $(64.8 \%)$ successfully extubated in the OR were male, whereas 529 of $849(62.3 \%)$ of those not successfully extubated in the OR were male.

prediction score based on only preoperative patient characteristics as the first step. Table 3 summarizes the preoperative factors that were independently associated with higher probability of OR extubation: younger age, lower body mass index, absence of diagnosis of chronic lung disease (CLD) and diabetes, surgical approaches other than full sternotomy, preoperative albumin more than $4 \mathrm{~g} / \mathrm{dL}$, isolated coronary artery bypass grafting (CABG), and elective operational status. Regression coefficients from the logistic model were translated into a simplified scoring algorithm, shown in the final column of Table 3; higher (positive) point values were assigned to those characteristics associated with a higher likelihood of successful OR extubation. When tested on the validation set of 398 patients (who were not included in the set that derived the score), the score demonstrated a strong relationship with probability of successful OR extubation (Figure 2, $A$ ). In particular, patients with scores equal to or less than zero had extremely low probabilities of successful OR extubation; as scores increase from +1 up to +7 , there is a progressive increase in the probability of successful OR extubation. The sensitivity and specificity of each threshold are shown in the last 2 columns of Table E2. The receiver operating characteristic curve showed that the model fitted well, with a $\mathrm{C}$-statistic of 0.833 on the derivation set and 0.820 on the validation set (Figure 3, A).

We repeated the same process to create a second version of the score using both preoperative and intraoperative characteristics; these results are summarized in the same format in Table 4. Table 4 shows the components of the score, which now includes the total dose of fentanyl and the use of multiple inhalational agents (isoflurane administered before and during cardiopulmonary bypass [CPB] and then switched over to desflurane or sevoflurane after weaning from $\mathrm{CPB}$ ). The use of lower doses of fentanyl and the use of multiple inhalational agents were associated with higher probability of OR extubation in addition to other preoperative variables. The performance of the score is illustrated in Figure 2, $B$, and described in Table E3. These results once again show that patients with scores below zero have minimal probability of successful OR extubation and then a progressive increase with scores in the positive range. The receiver operating characteristic curve for the model with both preoperative and intraoperative characteristics is shown in Figure 3, $B$; the C-statistic for this model was 0.877 on the derivation set and 0.868 on the validation set. The differences in C-statistic (Table E4) between the 2 scoring models ( 0.833 with model 1 [preoperative characteristics only] vs 0.877 with model 2 [preoperative + intraoperative characteristics] on the derivation set; 0.820 with model 1 vs 0.868 with model 2 on the validation set) were statistically significant $(P<.01$ for both the derivation and validation sets), suggesting that adding intraoperative characteristics improves the predictive ability of the model.

\section{DISCUSSION}

Extubation in the OR after adult cardiac operations is currently not widely practiced in cardiac surgery. The study aids clinical decision making by providing a validated prediction score for successful OR extubation.

Specifically, patients with a score less than zero (on the preoperative or preoperative + intraoperative score) are unlikely to be candidates for OR extubation, whereas patients with increasingly positive scores show progressively better probabilities of successful OR extubation. Patients with scores more than 5 are good candidates for extubation and should be encouraged within the comfort of the clinician. For scores between 0 and 5, successful OR extubation remains a possibility in some patients; however, a 
TABLE 2. Intraoperative characteristics of study population

\begin{tabular}{|c|c|c|c|}
\hline $\begin{array}{l}\text { Intraoperative } \\
\text { parameters }\end{array}$ & $\begin{array}{c}\text { ICU } \\
\text { extubation } \\
(\mathbf{N}=\mathbf{8 4 9})\end{array}$ & $\begin{array}{c}\text { Successful } \\
\text { OR extubation } \\
(\mathbf{N}=\mathbf{3 4 9})\end{array}$ & $\begin{array}{c}P \\
\text { value }\end{array}$ \\
\hline Operation & & & $<.001$ \\
\hline Isolated AVR & $110(13 \%)$ & $46(13 \%)$ & \\
\hline $\mathrm{AVR}+\mathrm{CABG}$ & $67(8 \%)$ & $4(1 \%)$ & \\
\hline $\mathrm{AVR}+\mathrm{MVR}$ & $5(0.6 \%)$ & $0(0 \%)$ & \\
\hline Isolated CABG & $298(35 \%)$ & $111(32 \%)$ & \\
\hline Isolated MVR & $29(3 \%)$ & $75(22 \%)$ & \\
\hline $\mathrm{MVR}+\mathrm{CABG}$ & $31(4 \%)$ & $7(2 \%)$ & \\
\hline Isolated MVR & $14(2 \%)$ & $8(2 \%)$ & \\
\hline $\mathrm{MVR}+\mathrm{CABG}$ & $6(0.7 \%)$ & $1(0.3 \%)$ & \\
\hline Other & $289(34 \%)$ & $97(28 \%)$ & \\
\hline Operative approach & & & $<.001$ \\
\hline Full conventional sternotomy* & $739(87 \%)$ & $168(48 \%)$ & \\
\hline Partial sternotomy & $55(7 \%)$ & $48(14 \%)$ & \\
\hline Right thoracotomy & $42(5 \%)$ & $108(31 \%)$ & \\
\hline Left thoracotomy & $6(0.7 \%)$ & $18(5 \%)$ & \\
\hline Minimally invasive & $5(0.6 \%)$ & $7(2 \%)$ & \\
\hline $\begin{array}{l}\text { Intra-aortic balloon } \\
\text { counter pulsation use }\end{array}$ & $49(6 \%)$ & $3(1 \%)$ & $<.001$ \\
\hline Any fentanyl & $841(99 \%)$ & $343(98 \%)$ & .308 \\
\hline Fentanyl dose $(\mu \mathrm{g})$ & $1000(0-3300)$ & $500(0-1750)$ & $<.001$ \\
\hline Any remifentanil & $25(3 \%)$ & $27(8 \%)$ & $<.001$ \\
\hline Remifentanil dose $(\mu \mathrm{g})$ & $0(0-10,000)$ & $0(0-8000)$ & $<.001$ \\
\hline Any dilaudid & $156(18 \%)$ & $148(42 \%)$ & $<.001$ \\
\hline Dilaudid dose (mg) & $0(0-9.2)$ & $0(0-4)$ & $<.001$ \\
\hline Any morphine & $22(3 \%)$ & $26(7 \%)$ & $<.001$ \\
\hline Morphine dose (mg) & $0(0-10)$ & $0(0-17)$ & $<.001$ \\
\hline Any intrathecal morphine & $37(4 \%)$ & $110(32 \%)$ & $<.001$ \\
\hline Any IV acetaminophen & $266(31 \%)$ & $244(70 \%)$ & $<.001$ \\
\hline Rocuronium dose (mg) & $150(0-1250)$ & $140(0-310)$ & $<.001$ \\
\hline Any midazolam & $835(98 \%)$ & $336(96 \%)$ & .027 \\
\hline Midazolam dose (mg) & $6.0(0-23)$ & $4.0(0-22)$ & $<.001$ \\
\hline Any etomidate & $504(59 \%)$ & $150(43 \%)$ & $<.001$ \\
\hline Etomidate dose (mg) & $12.0(0-140)$ & $0(0-200)$ & $<.001$ \\
\hline Any propofol & $263(31 \%)$ & $178(51 \%)$ & $<.001$ \\
\hline Propofol dose (mg) & $0(0-975)$ & $50(0-525)$ & $<.001$ \\
\hline Any ketamine & $2(0.2 \%)$ & $0(0 \%)$ & .364 \\
\hline Any lidocaine & $550(65 \%)$ & $286(82 \%)$ & $<.001$ \\
\hline Lidocaine dose (mg) & $100(0-2000)$ & $100(0-300)$ & $<.001$ \\
\hline Magnesium dose (g) & $4.0(0-120)$ & $4.0(0-13)$ & .174 \\
\hline No. of inotropes & & & $<.001$ \\
\hline 0 & $223(26 \%)$ & $105(30 \%)$ & \\
\hline 1 & $443(52 \%)$ & $208(60 \%)$ & \\
\hline $2+$ & $181(21 \%)$ & $34(10 \%)$ & \\
\hline No. of vasopressors & & & .834 \\
\hline 0 & $25(3 \%)$ & $9(3 \%)$ & \\
\hline 1 & $197(23 \%)$ & $86(25 \%)$ & \\
\hline $2+$ & $621(73 \%)$ & $252(72 \%)$ & \\
\hline Crystalloids (mL) & $1600(0-6004)$ & $1500(0-4846)$ & .363 \\
\hline Colloids (mL) & $500(0-5000)$ & $250(0-2350)$ & $<.001$ \\
\hline
\end{tabular}

(Continued)
TABLE 2. Continued

\begin{tabular}{|c|c|c|c|}
\hline $\begin{array}{c}\text { Intraoperative } \\
\text { parameters }\end{array}$ & $\begin{array}{c}\mathrm{ICU} \\
\text { extubation } \\
(\mathbf{N}=\mathbf{8 4 9}) \\
\end{array}$ & $\begin{array}{c}\text { Successful } \\
\text { OR extubation } \\
(\mathbf{N}=\mathbf{3 4 9})\end{array}$ & $\begin{array}{c}P \\
\text { value }\end{array}$ \\
\hline Any blood products administered & $162(19 \%)$ & $13(4 \%)$ & $<.001$ \\
\hline Any packed red blood cells & $121(14 \%)$ & $10(3 \%)$ & $<.001$ \\
\hline Any fresh-frozen plasma & $43(5 \%)$ & $0(0 \%)$ & $<.001$ \\
\hline Any platelets & $74(9 \%)$ & $5(1 \%)$ & $<.00$ \\
\hline Any cryoprecipitate & $21(3 \%)$ & $0(0 \%)$ & .003 \\
\hline $\begin{array}{l}\text { Inhalational agent } \\
\text { Isofluorane } \\
\text { Sevofluorane } \\
\text { Desflurane } \\
\text { Multiple agents }\end{array}$ & $\begin{array}{c}773(91 \%) \\
6(1 \%) \\
2(0.2 \%) \\
59(7 \%)\end{array}$ & $\begin{array}{c}245(70 \%) \\
3(1 \%) \\
4(1 \%) \\
88(25 \%)\end{array}$ & $<.001$ \\
\hline Postoperative LVEF (\%) & $50(0-80)$ & $55.0(15-90)$ & $<.001$ \\
\hline $\begin{array}{l}\text { Post-CPB right ventricular } \\
\text { dysfunction (moderate to severe) }\end{array}$ & $32(4 \%)$ & $4(1 \%)$ & .015 \\
\hline $\begin{array}{l}\text { Post-CPB left ventricular dysfunction } \\
\text { (moderate to severe) }\end{array}$ & $168(20 \%)$ & $24(7 \%)$ & $<.001$ \\
\hline Off-pump surgery & $99(12 \%)$ & $43(12 \%)$ & .748 \\
\hline Perfusion time (min) & $138(41-655)$ & $132(39-351)$ & .019 \\
\hline Crossclamp time (min) & $106(9-338)$ & $99.0(0-283)$ & .062 \\
\hline Surgery time $(\min )$ & $288(33-809)$ & $265(0-533)$ & $<.001$ \\
\hline Patient in to patient out OR time ( $\mathrm{min}$ ) & $386(118-900)$ & $373(115-773)$ & 013 \\
\hline
\end{tabular}

$I C U$, Intensive care unit; $O R$, operating room; $A V R$, Aortic valve replacement; $C A B G$ coronary artery bypass grafting; $M V R$, mitral valve replacement; $I V$, intravenous; $L V E F$, left ventricular ejection fraction; $C P B$, cardiopulmonary bypass. *Note that the percentages in this table are column percentages, eg, 168 of 349 patients $(48.1 \%)$ successfully extubated in the OR were those who underwent full sternotomy, whereas 739 of 849 patients $(87.0 \%)$ of those not successfully extubated in the OR underwent full sternotomy.

determination should be based on the experience and judgment of the clinician.

Extubation in the OR for suitable patients avoids discomfort from prolonged intubation and ventilation, avoids administration of unnecessary sedation medications, and may help to maintain better hemodynamics in an awake, spontaneously breathing patient. Previous studies have attempted to identify clinical factors that are associated with safe extubation in the OR in both selected and unselected cardiac surgical populations. ${ }^{24-31}$ Anesthesia drugs and techniques used vary among those clinical trials, but they all have a common objective of extubation at the end of surgery. Regional analgesia (thoracic epidural ${ }^{19}$ and paravertebral blocks ${ }^{30}$ ), total intravenous anesthesia ${ }^{32}$ (remifentanil, propofol), and short-acting inhalation anesthetics (sevoflurane and desflurane) ${ }^{31}$ have been used to facilitate recovery and extubation on the operating table. Longacting muscle relaxants, high dose of opioids (defined as fentanyl $>20 \mu \mathrm{g} / \mathrm{kg}$ ), and benzodiazepines such as midazolam are commonly avoided, especially in elderly patients. $^{23,24,26,27,31}$ Intrathecal morphine was used in patients undergoing thoracotomy in our study. We did not use other neuraxial blocks or total intravenous anesthesia. 
TABLE 3. Multivariate logistic regression model predicting successful operating room extubation (preoperative characteristics only)

\begin{tabular}{|c|c|c|c|c|c|c|}
\hline Preoperative patient characteristics & Beta & $95 \%$ CI & OR & $95 \% \mathrm{CI}$ & $P$ value & Score points \\
\hline \multicolumn{7}{|l|}{ Age, y } \\
\hline $40-49$ & 1.49 & $(0.86-2.12)$ & 4.45 & $(2.38-8.31)$ & $<.001$ & +3 \\
\hline $50-59$ & 1.02 & $(0.48-1.56)$ & 2.78 & $(1.63-4.74)$ & $<.001$ & +2 \\
\hline $60-69$ & 0.82 & $(0.32-1.31)$ & 2.27 & $(1.38-3.70)$ & .001 & +1 \\
\hline$>70$ (reference) & NA & (ref) & NA & (ref) & (ref) & \\
\hline \multicolumn{7}{|l|}{ BMI $\left(\mathrm{kg} / \mathrm{m}^{2}\right)$} \\
\hline$<25$ & 1.48 & $(0.79-2.16)$ & 4.39 & $(2.22-8.66)$ & $<.001$ & +3 \\
\hline $25-30$ & 1.10 & $(0.45-1.75)$ & 3.01 & $(1.57-5.77)$ & .001 & +2 \\
\hline $30-35$ & 0.72 & $(0.04-1.39)$ & 2.05 & $(1.04-4.00)$ & .036 & +1 \\
\hline$>35$ (reference) & NA & (ref) & NA & (ref) & (ref) & \\
\hline CLD & -0.79 & $(-1.53$ to -0.03$)$ & 0.46 & $(0.21-0.97)$ & .041 & -2 \\
\hline Diabetes & -0.39 & $(-0.81$ to 0.03$)$ & 0.67 & $(0.44-1.03)$ & .067 & -1 \\
\hline Non-full sternotomy (vs full sternotomy)* & 1.59 & $(1.11-2.06)$ & 4.89 & $(3.06-7.82)$ & $<.001$ & +3 \\
\hline Hypoalbuminemia (preoperative total albumin $<4 \mathrm{~g} / \mathrm{dL}$ ) & -0.96 & $(-1.62$ to -0.30$)$ & 0.38 & $(0.19-0.74)$ & .005 & -2 \\
\hline Peripheral vascular disease & -0.46 & $(-0.98$ to 0.07$)$ & 0.63 & $(0.37-1.07)$ & .089 & -1 \\
\hline Preoperative mediastinal radiation & -0.98 & $(-2.03$ to 0.08$)$ & 0.38 & $(0.13-1.08)$ & .069 & -2 \\
\hline \multicolumn{7}{|l|}{ Procedure } \\
\hline Isolated CABG (reference) & NA & (ref) & NA & (ref) & (ref) & \\
\hline Isolated AVR & -1.02 & $(-1.60$ to -0.34$)$ & 0.36 & $(0.18-0.71)$ & .003 & -2 \\
\hline Isolated MVR & -0.44 & $(-1.72$ to 0.84$)$ & 0.64 & $(0.17-2.33)$ & .501 & -1 \\
\hline Multiple operations & -1.29 & $(-2.10$ to -0.39$)$ & 0.27 & $(0.11-0.67)$ & .005 & -3 \\
\hline Other & -0.63 & $(-1.16$ to -0.09$)$ & 0.53 & $(0.31-0.92)$ & .023 & -1 \\
\hline Elective status (vs urgent) & 0.60 & $(0.15-1.04)$ & 1.82 & $(1.17-2.83)$ & .008 & +1 \\
\hline
\end{tabular}

$\overline{C I}$, Confidence interval; $O R$, operating room; $N A$, not available; $B M I$, body mass index; $C L D$, chronic lung disease; $C A B G$, coronary artery bypass grafting; $A V R$, Aortic valve replacement; $M V R$, mitral valve replacement. *Includes partial sternotomy, left thoracotomy, right thoracotomy, and minimally invasive surgery.

The individual anesthesiologist's practice guided the choice and dose of anesthesia drugs within the institution's fasttrack protocol (Table 1). Protocol-driven approaches dramatically improved early extubation performance. ${ }^{33}$ Probst and colleagues ${ }^{34}$ reported that a specialized postanesthesia care unit improved the time to extubation and transfer to a step-down unit without compromising patient safety after cardiac surgery. We cannot comment on the utility of postanesthesia care unit-based postoperative care for patients who are already extubated in the OR because all of our cardiac surgical patients in the current study recovered in the ICU by institutional policy. However, such a model is planned for future research.

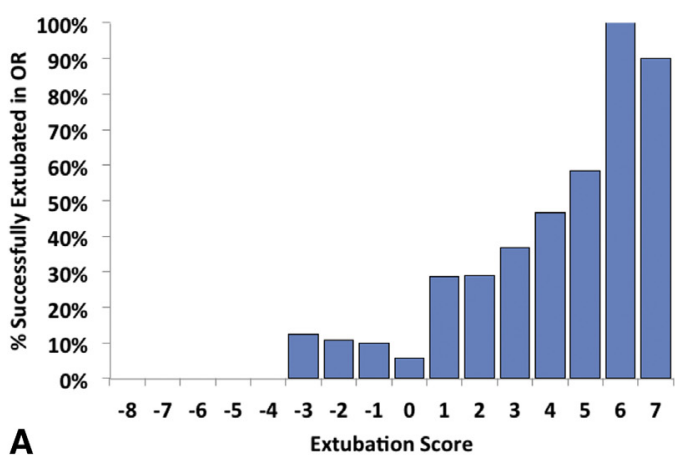

Rodriguez-Blanco and colleagues ${ }^{27}$ evaluated the factors associated with safe OR extubation in 272 consecutive patients after cardiac valve surgery. Maintenance of pulmonary perfusion and ventilation during $\mathrm{CPB}$ and lower duration of bypass predicted successful OR extubation in their multivariable model. None of the preoperative variables predicted OR extubation, and maintenance of perfusion and ventilation during $\mathrm{CPB}$ used in their study is not a common practice in cardiac surgery. Another study from the same group previously identified off-pump procedures, history of hypertension, preoperative ejection fraction greater than $30 \%$, and shorter surgical duration as predictors of successful OR

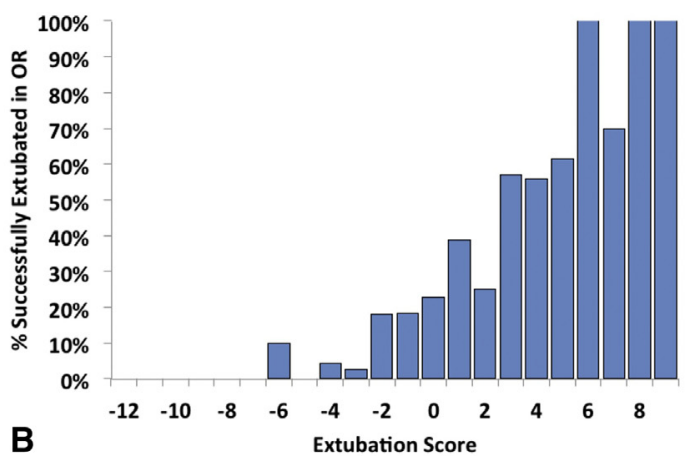

FIGURE 2. A, Percentage of patients successfully extubated in the OR in validation set $(\mathrm{n}=398)$ by score number 1 (preoperative characteristics only). B, Percentage of patients successfully extubated in the OR in validation set $(\mathrm{n}=398)$ by score number 2 (including intraoperative characteristics). OR, Operating room. 

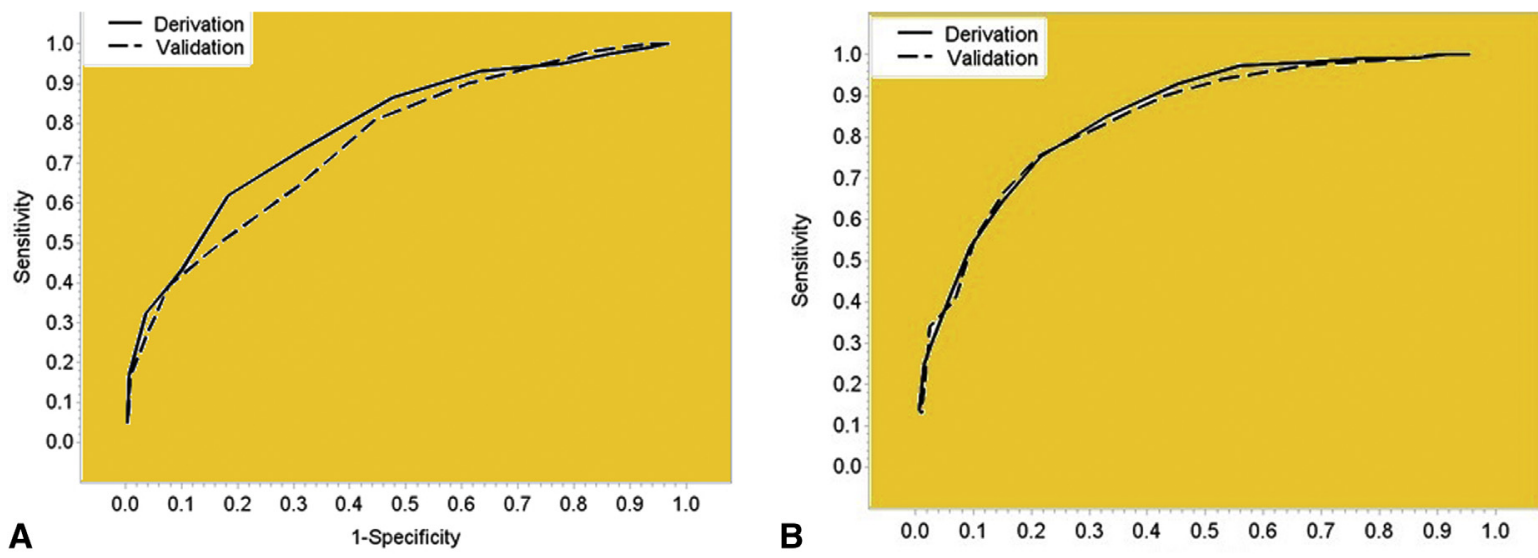

FIGURE 3. A, Receiver operating characteristic curves for model 1 (preoperative characteristics only). B, Receiver operating characteristic curves for model 2 (preoperative + intraoperative characteristics).

extubation in 372 patients undergoing all cardiac operations. ${ }^{26}$ Borracci and colleagues ${ }^{31}$ have shown that the Age, Creatinine, and Left Ventricular Ejection Fraction score was the only independent variable associated with OR extubation in 275 elderly patients ( $>70$ years) undergoing cardiac surgery. The majority of prior studies focused on a selected cardiac surgical population, involved a smaller sample size, and included a limited set of perioperative variables. ${ }^{26-32}$ The current study included all elective and urgent cardiac surgical procedures (on- and off-pump CABG, redo operations, isolated and multiple valve surgery and combined valve/CABG procedures, and sternotomy and thoracotomy approaches) representative of contemporary practice, and a comprehensive list of all preoperative and intraoperative variables from the STS database for association with OR extubation was examined. In addition to all STS variables, intraoperative anesthesia management data were included in the analysis unlike in previous studies, because anesthetic drug choice and dosages

TABLE 4. Multivariate logistic regression model predicting successful operating room extubation (including operative characteristics)

\begin{tabular}{|c|c|c|c|c|c|c|}
\hline Extubation predictors & Beta & $95 \% \mathrm{CI}$ & OR & $95 \% \mathrm{CI}$ & $P$ value & Score points \\
\hline \multicolumn{7}{|l|}{ Preoperative characteristics } \\
\hline \multicolumn{7}{|l|}{ Age, y } \\
\hline $40-49$ & 1.22 & $(0.41-2.02)$ & 3.39 & $(1.51-7.56)$ & .003 & +3 \\
\hline $50-59$ & 0.97 & $(0.26-1.67)$ & 2.63 & $(1.30-5.30)$ & .007 & +2 \\
\hline $60-69$ & 0.58 & $(-0.01$ to 1.23$)$ & 1.79 & $(0.93-3.42)$ & .080 & +1 \\
\hline$>70$ (reference) & NA & (ref) & NA & (ref) & (ref) & \\
\hline \multicolumn{7}{|l|}{ BMI $\left(\mathrm{kg} / \mathrm{m}^{2}\right)$} \\
\hline$<25$ & 1.22 & $(0.35-2.08)$ & 3.37 & $(1.42-7.99)$ & .006 & +3 \\
\hline $25-30$ & 0.81 & $(0.01-1.62)$ & 2.26 & $(1.01-5.04)$ & .047 & +2 \\
\hline $30-35$ & 0.68 & ( -0.17 to 1.53$)$ & 1.97 & $(0.84-4.60)$ & .119 & +1 \\
\hline$>35$ (reference) & NA & (ref) & NA & (ref) & (ref) & \\
\hline Diabetes & -1.18 & $(-1.79$ to -0.57$)$ & 0.31 & $(0.16-0.57)$ & $<.001$ & -2 \\
\hline Non-full sternotomy (vs full sternotomy)* & 1.84 & $(1.13-2.55)$ & 6.31 & $(3.12-12.77)$ & $<.001$ & +4 \\
\hline \multicolumn{7}{|l|}{ Procedure } \\
\hline Isolated CABG (reference) & NA & (ref) & NA & (ref) & (ref) & \\
\hline Isolated AVR & -1.96 & $(-2.91$ to -1.00$)$ & 0.14 & $(0.05-0.37)$ & $<.001$ & -4 \\
\hline Isolated MVR & -0.73 & $(-2.70$ to 1.25$)$ & 0.48 & $(0.06-3.48)$ & .471 & -1 \\
\hline Multiple operations & -1.57 & $(-2.62$ to -0.52$)$ & 0.21 & $(0.07-0.60)$ & .004 & -3 \\
\hline Other & -1.25 & $(-1.94$ to -0.56$)$ & 0.29 & $(0.14-0.57)$ & $<.001$ & -3 \\
\hline Elective status (vs urgent) & 0.56 & $(-0.07$ to 1.19$)$ & 1.75 & $(0.93-3.28)$ & .082 & +2 \\
\hline \multicolumn{7}{|l|}{ Intraoperative characteristics } \\
\hline Fentanyl dose (per $500 \mu \mathrm{g}$ ) & -1.20 & $(-1.61$ to -0.78$)$ & 0.30 & $(0.19-0.46)$ & $<.001$ & -2 \\
\hline Multiple inhalational agents & 1.33 & $(0.64-2.02)$ & 3.79 & $(1.91-7.51)$ & $<.001$ & +2 \\
\hline
\end{tabular}

$C I$, Confidence interval; $O R$, operating room; $N A$, not available; $B M I$, body mass index; $C A B G$, coronary artery bypass grafting; $A V R$, aortic valve replacement; $M V R$, mitral valve replacement. *Includes partial sternotomy, left thoracotomy, right thoracotomy, and minimally invasive surgery. 
can significantly influence emergence after cardiac surgery. To our knowledge, this is the most systematic study performed to identify preoperative and intraoperative factors that are independently associated with successful OR extubation in a generalized, unselected cardiac surgery population.

In a large, nonrandomized, observational study, Chamchad and colleagues ${ }^{24}$ evaluated several perioperative variables (demographic data, preoperative medical status, left ventricular function, operative events, and procedure times) and identified predictors of failed OR extubation. The anesthesia technique used was similar to that used in the current study (propofol, rocuronium, intermittent fentanyl, and inhalational anesthetics). In the current study, we included additional important intraoperative variables in the model, especially anesthetic drug doses, transfusions, fluids administered, inotropic and vasopressor requirement, and postbypass ventricular function. Patients extubated in the OR received lower doses of fentanyl and shorter-acting inhalational anesthetics (desflurane and sevoflurane) in the postbypass period. Few anesthesiologists prefer administration of isoflurane before, during, and after bypass, whereas others switched to sevoflurane during the postbypass period in an effort to facilitate extubation in the OR. We attempted to determine whether such changes in practice made any difference in extubating patients in the OR. Switching to shorter-acting sevoflurane seems to facilitate extubation in the OR, although we cannot rule out bias from the anesthesia providers that they switched over to sevoflurane in patients with a higher likelihood of successful OR extubation.

Adding intraoperative variables improved the performance of the OR extubation prediction score in this study. Clinicians should make decisions regarding OR extubation at the end of surgery after evaluation of preoperative patient characteristics, intraoperative anesthetic drug doses, and surgical course. This complex interplay of factors is the basis for formulating a scoring system representing all significant perioperative variables to guide the decision-making process for OR extubation. The scores were developed with a derivation dataset of 800 patients and demonstrated good predictive ability on a separate validation dataset of 398 patients. The scores behave particularly well at the extremes, identifying subgroups of patients with very low probability of successful OR extubation (patients with scores $\leq 0$ ) and patients with very high probability of successful OR extubation (patients with scores near the top end of the range). Clinician experience and preference will still play a large role in decision making for patients in the middle zone of the scoring system.

Preexisting hypoalbuminemia was found to be an independent factor negatively associated with OR extubation. Low serum albumin could be a proxy for concurrent nutritional status and clinical frailty. Likewise, diabetes may reflect end-organ dysfunction, atherosclerotic disease, and other associated comorbid conditions. CLD and mediastinal radiation were predictors of delayed extubation when only preoperative characteristics were considered in the model, but they were not significant when intraoperative factors were included in the model. CLD may be of variable severity, and in this study, less than $5 \%$ of population with CLD had severe enough disease to require home oxygen therapy.

Patients undergoing full conventional sternotomy were extubated less often than patients undergoing cardiac operations performed through other approaches, such as thoracotomy. This may be explained by the use of minimally invasive surgical incisions and intrathecal morphine for analgesia in patients undergoing thoracotomy. In univariable analyses of the derivation set, the patients undergoing isolated mitral valve repair/replacement (MVRR) had higher successful OR extubations (65\%) than the patients undergoing isolated CABG $(27 \%)$. On further examination of each individual covariate as a potential confounder, we determined that the addition of "elective versus urgent" had the greatest effect on these estimates, most likely because of the isolated MVRR procedures that were elective, which "flipped" the direction of the univariable effect. Patients undergoing isolated MVRR are more likely to be extubated in the OR than those undergoing isolated AVR procedures. This may simply reflect the presence of more severe comorbidities in patients with aortic stenosis because of relatively advanced age at the time of surgery.

\section{Study Limitations}

The limitations of the present study include those of all large single-center retrospective studies. First, although all cardiac surgical patients during this period were treated in the same protocolized extubation pathway, anesthesiologist- or surgeon-specific preferences for OR extubation could not be controlled for. Second, logistic factors limiting OR extubation, such as procedures extending into late evening and pressure to start another operation in the same room, could have played a role for some patients in this retrospective study. Third, given the small number of patients $(n=5)$ requiring reintubation, this study was not powered to identify patient characteristics associated with initial OR extubation and subsequent early reintubation. Three patients who underwent minimally invasive MVRR required reintubation for hypoventilation and hypercapnic respiratory failure shortly after arrival in the ICU. Postoperative neurologic dysfunction after off-pump CABG and acute pulmonary edema after the MAZE procedure were the other 2 reasons for reintubation in this study. Finally, this cohort was split into groups to perform a derivation set and a test set for validation. Although the score performed well in the 
validation set, a large prospective validation study with patients recruited from multiple centers will be ideal to prove the utility of this prediction scoring system.

\section{CONCLUSIONS}

We report independent factors associated with successful OR extubation after adult cardiac operations. A simplified preoperative and operative scoring system to predict successful OR extubation is outlined. This scoring system may be a reasonable reference point to aid programs with commencement or refinement of their practice of immediate $\mathrm{OR}$ extubation.

\section{Conflict of Interest Statement}

Authors have nothing to disclose with regard to commercial support.

\section{References}

1. Cheng DC, Karski J, Peniston C, Raveendran G, Asokumar B, Carroll J, et al. Early tracheal extubation after coronary artery bypass surgery reduces costs and improves resource use: a prospective, randomized, controlled trial. Anesthesiology. 1996;85:1300-10.

2. Cheng DC, Wall C, Djaiani G, Peragallo RA, Carroll J, Li C, et al. Randomized assessment of resource use in fast-track cardiac surgery 1-year after hospital discharge. Anesthesiology. 2003;98:651-7.

3. Velasco FT, Tarlow LS, Thomas SJ. Economic rationale for early extubation. J Cardiothorac Vasc Anesth. 1995;9:2-9.

4. Cheng DC. Early extubation after cardiac surgery decreases intensive care unit stay and cost. J Cardiothorac Vasc Anesth. 1995;9:460-4.

5. Avrom KV, Emery RW, Peterson RJ, Schwartz M. Cost-effectiveness and predictors of early extubation. Ann Thorac Surg. 1995;60:127-32.

6. Trouillet JL, Combes A, Vaissier E, Luyt CE, Quattara A, Pavie A, et al. Prolonged mechanical ventilation after cardiac surgery: outcome and predictors. $J$ Thorac Cardiovasc Surg. 2009;138:948-53.

7. Reis J, Mota JC, Ponce P, Costa-Pereira A, Guerreiro M. Early extubation does not increase complication rates after coronary artery bypass graft surgery with cardiopulmonary bypass. Eur J Cardiothorac Surg. 2002;21:1026-30.

8. Silbert BS, Santamaria JD, O'Brien JL, Blyth CM, Kelly WJ, Molnar RR. Early extubation following coronary artery bypass surgery: a prospective randomized controlled trial. The Fast Track Cardiac Care Team. Chest. 1998;113:1481-8.

9. Berry P, Thomas S, Mahon S, Jackson M, Fox MA, Fabri B, et al. Myocardial ischemia after coronary artery bypass grafting: early vs late extubation. $\mathrm{Br} J$ Anaesth. 1998;80:20-5.

10. Myles PS, Daly DJ, Djaiani G, Lee A, Cheng DCH. A systematic review of the safety and effectiveness of fast-track cardiac anesthesia. Anesthesiology. 2003; 99:982-7.

11. Alhan C, Toraman F, Karabulut EH, Racan S, Dagdelen S, Eren N, et al. Fast track recovery of high risk coronary artery bypass surgery patients. Eur J Cardiothorac Surg. 2003;23:678-83.

12. Cheng DC, Karski J, Peniston C, Asokumar B, Raveendran G, Carroll J, et al. Morbidity outcome in early versus conventional tracheal extubation after coronary artery bypass grafting: a prospective randomized controlled trial. J Thorac Cardiovasc Surg. 1996;112:755-64.

13. Quasha AL, Loeber W, Feeley TW, Ullyot DJ, Roizen MF. Postoperative respiratory care: a controlled trial of early and late extubation following coronary artery bypass grafting. Anesthesiology. 1980;52:135-41.

14. Reddy SL, Grayson AD, Griffiths EM, Pulan DM, Rashid A. Logistic risk model for prolonged ventilation after adult cardiac surgery. Ann Thorac Surg. 2007;84: 528-36.

15. Totonchi Z, Baazm F, Chitsazan M, Seifi S, Chitsazan M. Predictors of prolonged mechanical ventilation after open heart surgery. J Cardiovasc Thorac Res. 2014; 6:211-6.
16. Saleh HZ, Shaw M, Al-Rawi O, Yates J, Pullan DM, Chalmers JAC et al. Outcomes and predictors of prolonged ventilation in patients undergoing elective coronary surgery. Interact Cardiovasc Thorac Surg. 2012;15: 51-6.

17. Wang C, Zhang GX, Lu FL, Li BL, Zou LJ, Han L, et al. A local risk prediction model for prolonged ventilation after adult heart valve surgery in a Chinese single center. Heart Lung. 2013;42:13-8.

18. Royse CF, Royse AG, Soeding PF. Routine immediate extubation after cardiac operation: a review of our first 100 patients. Ann Thorac Surg. 1999;68: 1326-9.

19. Djaiani GN, Ali M, Heinrich L, Bruce J, Carroll J, Karski J, et al. Ultra-fast-track anesthetic technique facilitates operating room extubation in patients undergoing off-pump coronary revascularization surgery. J Cardiothorac Vasc Anesth. 2001; 15:152-7.

20. Sullivan BL. Con: early extubation in the operating room following cardiac surgery. Semin Cardiothorac Vasc Anesth. 2012;16:187-9.

21. Siliciano D. Con: early extubation is not preferable to late extubation in patients undergoing cardiac surgery. J Cardiothorac Vasc Anesth. 1992;6: 494-8.

22. Badhwar V, Esper SA, Brooks M, Mulukutla S, Hardison R, Mallios D, et al. Extubating in the operating room after adult cardiac surgery safely improves outcomes and lowers costs. J Thorac Cardiovasc Surg. 2014;148: 3101-9.

23. Sullivan LM, Massaro JM, D'Agostino RB Sr. Presentation of multivariate data for clinical use. Stat Med. 2004;23:1631-60.

24. Chamchad D, Horrow JC, Nachamchik L, Sutter FP, Samuels LE, Trace CL, et al The impact of immediate extubation in the operating room after cardiac surgery on intensive care and hospital lengths of stay. J Cardiothorac Vasc Anesth. 2010; 24:780-4.

25. Dorsa AG, Rossi AI, Thierer J, Lupiañez B, Vrancic JM, Vaccarino GN, et al. Immediate extubation after off-pump coronary artery bypass graft surgery in 1,196 consecutive patients: feasibility, safety and predictors of when not to attempt it. $J$ Cardiothorac Vasc Anesth. 2011;25:431-6.

26. Rodriguez Blanco YF, Candiotti K, Gologorsky A, Tang F, Giquel J, Barron ME, et al. Factors which predict safe extubation in the operating room following cardiac surgery. J Card Surg. 2012;27:275-80.

27. Rodriguez-Blanco YF, Carvalho EM, Gologorsky A, Lo K, Salerno TA, Gologorsky E. Factors associated with safe extubation in the operating room after on-pump cardiac valve surgery. J Card Surg. 2016;31:274-81.

28. Miller JW, Vu D, Chai PJ, Kreutzer J, Hossain MM, Jacobs JP, et al. Patient and procedural characteristics for successful and failed immediate tracheal extubation in the operating room following cardiac surgery in infancy. Paediatr Anaesth. 2014;24:830-9.

29. Garg R, Rao S, John C, Reddy C, Hegde R, Murthy K, et al. Extubation in the operating room after cardiac surgery in children: a prospective observational study with multidisciplinary coordinated approach. J Cardiothorac Vasc Anesth. 2014;28:479-87.

30. Neuburger PJ, Chacon MM, Luria BJ, Manrique-Espinel AM, Ngai JY, Grossi EA, et al. Does paravertebral blockade facilitate immediate extubation after totally endoscopic robotic mitral valve repair surgery? Innovations (Phila). 2015;10:96-100.

31. Borracci RA, Ochoa G, Ingino CA, Lebus JM, Grimaldi SV, Gambetta MX Routine operation theatre extubation after cardiac surgery in the elderly. Interact Cardiovasc Thorac Surg. 2016;22:627-32.

32. Straka Z, Brucek P, Vanek T, Votava J, Widimsky P. Routine immediate extubation for off-pump coronary artery bypass grafting without thoracic epidural analgesia. Ann Thorac Surg. 2002;74:1544-7.

33. Fitch ZW, Debesa O, Ohkuma R, Duquaine D, Steppan J, Schneider EB, et al. A protocol-driven approach to early extubation after heart surgery. J Thorac Cardiovasc Surg. 2014;147:1344-50.

34. Probst S, Cech C, Haentschel D, Scholz M, Ender J. A specialized post anaesthetic care unit improves fast-track management in cardiac surgery: a prospective randomized trial. Crit Care. 2014;18:468.

Key Words: adult cardiac surgery, extubation, perioperative management 
TABLE E1. Medications and dose range to facilitate early or operating room extubation

\section{Midazolam 1-4 mg}

Fentanyl 250-1000 $\mu \mathrm{g}$

Etomidate $10-20 \mathrm{mg}$

Propofol 50-200 mg

Ketamine 50-100 mg

Inhalational anesthetics 0.5-1.5 MAC titrated to bispectral index (40-60) and hemodynamics (isoflurane before and during $\mathrm{CPB}$ but sevoflurane or desflurane substituted isoflurane after termination of CPB by anesthesiologist's preference)

Remifentanil 0.1-0.4 $\mu \mathrm{g} / \mathrm{kg} / \mathrm{min}$ until skin closure

Hydromorphone 0.4-2 mg at the end of the case titrated to patient's pain Acetaminophen $1 \mathrm{~g}$ IV after termination of $\mathrm{CPB}$

$M A C$, Minimum alveolar concentration; $C P B$, cardiopulmonary bypass; $I V$, intravenously.

TABLE E2. Percentage of patients successfully extubated in operating room in validation set $(n=398)$ by score number 1 (preoperative characteristics only)

\begin{tabular}{|c|c|c|c|c|}
\hline \multirow[b]{2}{*}{ Score points } & \multicolumn{3}{|c|}{ n $(\%)$} & \multirow[b]{2}{*}{ Specificity $(\%)^{*}$} \\
\hline & No. of patients & Successful OR extubation & Sensitivity $(\%)^{*}$ & \\
\hline-8 & 1 & $0(0.0)$ & 100.0 & 0.0 \\
\hline-7 & 0 & NA & 100.0 & 0.0 \\
\hline-6 & 2 & $0(0.0)$ & 100.0 & 0.7 \\
\hline-5 & 2 & $0(0.0)$ & 100.0 & 2.2 \\
\hline-4 & 8 & $0(0.0)$ & 99.2 & 5.5 \\
\hline-3 & 24 & $3(12.5)$ & 97.6 & 12.8 \\
\hline-2 & 28 & $3(10.7)$ & 94.4 & 20.9 \\
\hline-1 & 40 & $4(10.0)$ & 92.0 & 36.3 \\
\hline 0 & 52 & $3(5.8)$ & 84.0 & 52.7 \\
\hline 1 & 49 & $14(28.6)$ & 65.6 & 67.0 \\
\hline 2 & 62 & $18(29.0)$ & 52.8 & 81.3 \\
\hline 3 & 49 & $18(36.7)$ & 40.8 & 91.2 \\
\hline 4 & 30 & $14(46.7)$ & 26.4 & 95.2 \\
\hline 5 & 24 & $14(58.3)$ & 13.6 & 98.5 \\
\hline 6 & 17 & $17(100.0)$ & 4.0 & 99.3 \\
\hline 7 & 10 & $9(90.0)$ & 0.0 & 100.0 \\
\hline
\end{tabular}

$O R$, Operating room; $N A$, not available. *Sensitivity and specificity calculated as follows: For each score threshold, we assume that all patients greater than or equal to the corresponding score are predicted to have successful OR extubations. In this case, a "positive" represents an OR extubation, so a high sensitivity means that a high proportion of the patients who were extubated successfully in the OR are correctly identified; a "negative" represents a patient who was not successfully extubated in the OR, so a high specificity means that a high proportion of patients who could not be extubated in the OR are correctly identified. Scores at the low end of the spectrum have high "sensitivity" and low "specificity" because those thresholds would suggest that all patients should be extubated in the OR; scores at the high end of the spectrum have low "sensitivity" and high "specificity" because they take the opposing extreme, suggesting that virtually no one should be extubated in the OR (thereby resulting in lower "false-positive" rates, at the cost of suggesting that many patients who were successfully extubated in the OR should not have been). The decision about which threshold offers the best combination is likely a physician-specific preference; however, the very high sensitivity of scores at or below zero indicates that at minimum, the score functions well to identify patients with low scores as having a minimal chance of successful OR extubation; likewise, patients with high scores tended to have a good probability of successful OR extubation. 
TABLE E3. Percentage of patients successfully extubated in operating room in validation set $(n=398)$ by score number 1 (including operative characteristics)

\begin{tabular}{|c|c|c|c|c|}
\hline \multirow[b]{2}{*}{ Score points } & \multicolumn{3}{|c|}{ n $(\%)$} & \multirow[b]{2}{*}{ Specificity $(\%) *$} \\
\hline & No. of patients & Successful OR extubation & Sensitivity $(\%)^{*}$ & \\
\hline-12 & 1 & $0(0.0)$ & 100.0 & 0.3 \\
\hline-11 & 1 & $0(0.0)$ & 100.0 & 0.7 \\
\hline-10 & 2 & $0(0.0)$ & 100.0 & 1.4 \\
\hline-9 & 2 & $0(0.0)$ & 100.0 & 2.1 \\
\hline-8 & 3 & $0(0.0)$ & 100.0 & 3.1 \\
\hline-7 & 9 & $0(0.0)$ & 100.0 & 6.3 \\
\hline-6 & 10 & $1(10.0)$ & 99.1 & 9.4 \\
\hline-5 & 31 & $0(0.0)$ & 99.1 & 20.2 \\
\hline-4 & 23 & $1(4.4)$ & 98.2 & 27.9 \\
\hline-3 & 38 & $1(2.6)$ & 97.3 & 40.8 \\
\hline-2 & 39 & $7(17.9)$ & 91.0 & 51.9 \\
\hline-1 & 44 & $8(18.2)$ & 83.8 & 64.5 \\
\hline 0 & 44 & $10(22.7)$ & 74.8 & 76.3 \\
\hline 1 & 31 & $12(38.7)$ & 64.0 & 82.9 \\
\hline 2 & 28 & $7(25.0)$ & 57.7 & 90.2 \\
\hline 3 & 21 & $12(57.1)$ & 46.8 & 93.4 \\
\hline 4 & 25 & $14(56.0)$ & 34.2 & 97.2 \\
\hline 5 & 13 & 8 (61.5) & 27.0 & 99.0 \\
\hline 6 & 20 & $20(100.0)$ & 9.0 & 99.0 \\
\hline 7 & 10 & $7(70.0)$ & 2.7 & 100.0 \\
\hline 8 & 1 & $1(100.0)$ & 1.8 & 100.0 \\
\hline 9 & 2 & $2(100.0)$ & 0.0 & 100.0 \\
\hline \multicolumn{5}{|c|}{$\begin{array}{l}O R \text {, Operating room. *Sensitivity and specificity calculated as follows: for each score threshold, we assume that all patients greater than or equal to the corresponding score are } \\
\text { predicted to have successful OR extubations. In this case, a "positive" represents an OR extubation, so a high sensitivity means that a high proportion of the patients who were } \\
\text { extubated successfully in the OR are correctly identified; a "negative" represents a patient who was not successfully extubated in the OR, so a high specificity means that a high } \\
\text { proportion of patients who could not be extubated in the OR are correctly identified. Scores at the low end of the spectrum have high "sensitivity" and low "specificity" because } \\
\text { those thresholds would suggest that all patients should be extubated in the OR; scores at the high end of the spectrum have low "sensitivity" and high "specificity" because they } \\
\text { take the opposing extreme, suggesting that virtually no one should be extubated in the OR (thereby resulting in lower "false-positive" rates, at the cost of suggesting that many } \\
\text { patients who were successfully extubated in the OR should not have been). The decision about which threshold offers the best combination is likely a physician-specific pref- } \\
\text { erence; however, the high sensitivity of scores at or below zero indicates that at minimum, the score functions well to identify patients with low scores as having minimal chance of } \\
\text { successful OR extubation; likewise, patients with high scores tended to have a good probability of successful OR extubation. }\end{array}$} \\
\hline
\end{tabular}

TABLE E4. Area under the curve (c-statistics) for each model

\begin{tabular}{lcc}
\hline \multicolumn{1}{c}{ Model } & Derivation set & Validation set \\
\hline $\begin{array}{l}\text { Preoperative characteristics } \\
\text { only }\end{array}$ & 0.833 & 0.820 \\
Preoperative + intraoperative & 0.877 & 0.868 \\
\hline
\end{tabular}

